\title{
Associação entre a altura uterina e o peso fetal nas gestantes de baixo risco
}

\author{
Association between uterine heigh and fetal weight in low-risk pregnant women
}

Asociación entre la altura uterina y el peso fetal em mujeres con gestación de bajo riesgo

Gabriela Pacheco de Assis ${ }^{1}$, Luisa Paschoal Prudente ${ }^{1}$, Ana Laura Mesquita Teixeira ${ }^{1}$, Isabella Oliveira Rocha1', Giovana Bellettato Reche ${ }^{1}$, Gabriel Duque Pannain², Juliana Barroso Zimmermmann ${ }^{1,2 *}$.

\section{RESUMO}

Objetivo: Avaliar o crescimento fetal mensurado pela altura uterina e sua associação com o peso fetal estimado ao ultrassom. Métodos: Estudo de corte transversal de pacientes atendidas pelo serviço de Obstetrícia, durante o período de 2008 a 2021. Foram usados dados de anamnese, do exame físico, da avaliação ultrassonográfica do crescimento fetal em todos os trimestres e da medida uterina de gestantes de baixo risco obstétrico. Resultados: Foram estudadas 883 gestantes, com a média de idade de 25,5 $\pm 7,29$ anos. As alturas uterinas foram divididas em percentis de acordo com intervalos das semanas gestacionais e foram assim identificadas em centímetros: $17,0 \pm 2,59$ (16-20 semanas; 4o mês), 21,0 \pm 2,27 (20-24 semanas;

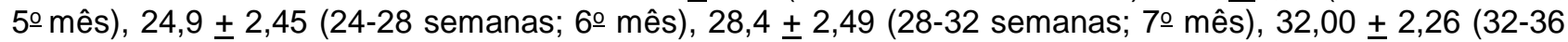
semanas; 8ㅇ mês) e 34,00 $\pm 12,00$ ( $>36$ semanas; 9 o mês). A altura uterina se associou ao peso fetal $(p<0,05)$. Conclusão: Identificou-se que a curva de altura uterina x peso fetal $x$ idade gestacional pode ser metodologia de uso em acompanhamento de gestantes de baixo risco, já que o peso fetal aumenta com a idade gestacional.

Palavras-chave: Peso fetal, Restrição de crescimento fetal, Macrossomia fetal.

\begin{abstract}
Objective: Evaluate fetal growth measured by uterine height and its association with fetal growth deviations and patient data. Methods: Cross-sectional study of patients attended by the obstetrics services during the period from 2008 to 2021. Data from anamnesis, physical examination, ultrasound assessment of fetal growth in all trimesters and uterine measurement of low-risk pregnant women were used in the study. Results: 883 pregnant women with an average age of $25.5 \pm 7,29$. Uterine heights were divided into percentiles according to gestational week intervals and were thus identified in centimeters: $17.0+2.59$ (16-20 weeks; 4th month), $21.0+2.27$ (20-24 weeks; 5th month), $24.9+2.45$ (24-28 weeks; 6th month), $28.4+2.49$ (28-32 weeks; 7th month), $32.00+2.26$ (32-36 weeks; 8th month) and $34.00+12.00$ (> 36 weeks; 9th month). Uterine height was associated with fetal weight $(p<0.05)$. Conclusion: We identified that the curve of uterine height $X$ fetal weight $X$ gestational age can be used in monitoring low-risk pregnant women, since fetal weight increases with gestational age.
\end{abstract}

Keywords: Fetal weight, Fetal growth restriction, Fetal macrosomia.

\section{RESUMEN}

Objetivo: Evaluar el crecimiento fetal medido por la altura uterina y su asociación con el peso fetal y los datos del paciente. Métodos: Estudio corte transversal de pacientes atendidos por el servicio de obstetricia durante el periodo 2008-2021. Durante el estudio se utilizó “data from anamnesis" examinación física, revisión de ultrasonido del crecimiento fetal en todos los trimestres y medición uterina de mujeres embarazadas con bajo riesgo que participaron en el estudio. Resultados: Se estudiaron 883 casos de mujeres embarazadas, con una edad promedio de $25.5 \pm 7,29$. Las alturas uterinas se dividieron en percentiles de acuerdo con los intervalos de la semana gestacional y, por lo tanto, se identificaron en centímetros: $17.0 \pm 2.59$ (16-20

\footnotetext{
${ }^{1}$ Faculdade de Medicina de Barbacena, Barbacena - MG. *E-mail: julianabz@uol.com.br
}

2 Universidade Federal de Juiz de Fora, Juiz de Fora - MG.

SUBMETIDO EM: 5/2021

ACEITO EM: 6/2021

PUBLICADO EM: 6/2021 
semanas; $4^{\circ}$ mes), $21.0 \pm 2.27$ (20-24 semanas; $5^{\circ}$ mes), $24.9 \pm 2.45$ (24- 28 semanas; $6^{\circ}$ mes), 28,4 $+2,49$ (28-32 semanas; 7ํㅡㄹ altura uterina se asoció con el peso fetal $(p<0,05)$. Conclusión: Identificamos que la curva de altura uterina $X$ peso fetal $X$ edad gestacional puede ser utilizada para monitorear mujeres embarazadas de bajo riesgo, ya que el peso fetal incrementa con la edad gestacional.

Palabras clave: Peso fetal, Restricción del crecimiento fetal, Macrosomía fetal.

\section{INTRODUÇÃO}

O crescimento fetal depende de uma série de fatores, sejam genéticos, hormonais, nutricionais e até ambientais. Pode-se dizer que a função ótima placentária depende de um remodelamento vascular eficiente, com criação de um fluxo vascular de baixa resistência, responsável por permitir a nutrição do feto e desenvolvimento intraútero. Entretanto, nem sempre isso acontece. Algumas vezes, fatores maternos, fetais ou ambientais podem influenciar nos desvios de crescimento fetal (CROCKART IC, et al., 2021; MOREIRA NETO AR, et al., 2011).

A Restrição do Crescimento Fetal (RCF) é a segunda principal causa de morbidade e mortalidade perinatal, sendo superada apenas pela prematuridade, de forma que a mortalidade perinatal aumenta de 6 a 10 vezes em recém-nascidos com RCF (CROCKART IC, et al., 2021; DETER RL, et al., 2021; MOREIRA NETO AR, et al., 2011).

Pode-se dizer que há RCF quando o feto não atinge todo o seu potencial genético de crescimento ou seja, quando se encontra abaixo do percentil 10 para a idade gestacional. Vários fatores são citados como responsáveis, dentre eles, condições sociais e econômicas, sendo, por isso, mais comuns em países em desenvolvimento; as doenças maternas (síndromes hipertensivas, colagenoses, diabetes mellitus) e placentárias (insuficiência placentária) (ANTONELLI JDS, et al., 2018). As principais consequências da RCF são as complicações neurológicas, policitemia, hipoglicemia, aspiração meconial e morte. Para a vida adulta, as repercussões estão associadas ao desenvolvimento de diabetes e de doenças cardiovasculares, especialmente pela herança epigenética (MOREIRA NETO AR, et al., 2011).

Por outro lado, o feto pode também apresentar crescimento excessivo, sendo chamado de macrossômico. A macrossomia é definida quando o peso fetal estimado é maior que $4.000 \mathrm{~g}$, variando de 0,4 a $10 \%$ e pode determinar uma série de complicações, especialmente para o parto, como a distócia de ombros, lesão do plexo braquial, fraturas de clavícula e fraturas de úmero e até complicações neurológicas graves pelo trauma durante o parto. Está associada ao diabetes gestacional, pós-datismo, antecedente de feto macrossômico e peso materno (CALDERON IMP e RUDGE MVC, 2006).

O diagnóstico destes desvios pode ser realizado pela avaliação clínica através do cuidado pré-natal, além das medidas ultrassonográficas habituais. O método clínico mais utilizado na prática obstétrica diária para acompanhamento do crescimento fetal é a medida da altura uterina, realizada através da fita métrica, medindo a distância $(\mathrm{cm})$ entre a sínfise púbica ao fundo uterino. O resultado dessa medida é colocado em curvas para avaliação do crescimento fetal de acordo com a idade gestacional (ZIMMERMMANN JB, et al., 2018). Uma curva muito utilizada é a de Belizán J, et al. (1978) da Argentina, mas também existem as curvas de Quaranta $P$, et al (1981) da Inglaterra, de Taylor $P$, et al (1984) na Austrália.

No Brasil, utiliza-se uma curva realizada a partir de uma amostra de gestantes uruguaias. $\mathrm{O}$ grande problema é que essas curvas são baseadas em estudos internacionais e extrapolar para a nossa população poderia ser considerado um viés, já que as condições genéticas, raciais e nutricionais são importantes variáveis no crescimento fetal (CROCKART IC, et al., 2021; CECATTI JG, et al., 2000). Em 2001, Martinelli $\mathrm{S}$, et al. (2011) escreveu uma nova curva com população brasileira, identificando crescimento de 0,7 $\mathrm{cm} /$ semana na altura uterina, entretanto sendo o Brasil um país muito grande, com diferenças regionais, as curvas de crescimento podem variar de acordo com a população estudada (CECATTI JG, et al., 2000; FREIRE DMC, et al., 2006; BARINI R, et al., 1997). 
Outra possibilidade de avaliação do crescimento fetal é a determinação do Peso fetal estimado (PFE) pela Ultrassonografia (USG). Várias fórmulas foram criadas para estimar o peso fetal a partir de parâmetros biométricos, podendo incluir o diâmetro bi parietal, a medida do fêmur e a circunferência abdominal (SHARP A, et al., 2018; BLUE NR, et al., 2018).

A escolha do melhor parâmetro para avaliar o crescimento fetal é controversa. Estudo realizado na Turquia, comparando o peso fetal estimado ao ultrassom, com a medida da altura uterina e a circunferência abdominal materna identificou que todos os três métodos superestimaram estatisticamente o peso ao nascer (ZAHRAN $M$, et al., 2015). Dessa forma, apesar dos avanços na USG, tanto a medida da altura uterina como as ultrassonográficas, apresentam-se limitadas para predizer o peso neonatal (CURTI A, et al., 2014; ROBERT PETER J, et al., 2015). Entretanto, estudos recentes já incluem marcadores inflamatórios para esse diagnóstico, o que apesar de aumentar a sensibilidade, incrementa o custo, sendo impossível de ser realizado em muitos locais (GONZALÉS-FERNANDES D, et al., 2021).

Vivemos em um país em desenvolvimento, com recursos escassos e a utilização de ultrassonografia obstétrica rotineira em população de baixo risco significa um gasto que muitas cidades não podem arcar, sem contar que existe a zona rural e aquelas áreas com acesso difícil e sem ultrassonografia disponível. Além disso, no Brasil, o Ministério da Saúde (MS) recomenda a utilização da curva de crescimento da altura uterina baseado em uma amostra de mulheres uruguaias, a partir do Centro Latino-Americano de Perinatologia (CLAP), considerando os percentis 10 e 90, os limites inferior e superior (BRASIL, 2012), que não representa a nossa população. Desta forma, poderíamos estar considerando como normal, parâmetros inadequados para a nossa população, o que deixaria a assistência materno-fetal prejudicada.

Baseado no exposto, objetivou-se construir uma curva de normalidade para a altura uterina, em função da idade gestacional e do peso fetal estimado pelo ultrassom, em gestantes com idade gestacional confiável, de baixo risco obstétrico, sem fatores associados para os desvios de crescimento, no estado de Minas Gerais.

\section{MÉTODOS}

Trata-se de uma coorte histórica onde foram estudadas pacientes oriundas do serviço de Obstetrícia da Faculdade de Medicina no período de setembro de 2008 até a presente data. Todas as pacientes foram submetidas à anamnese e exame físico obstétrico, bem como a propedêutica pré-natal habitual (FEBRASGO, 2014).

Atualmente existem 6700 gestantes em nossa cidade e o serviço atende em média 300 gestantes/mês. Considerando a frequência de desvios de crescimento fetal da ordem de $20 \%$, erro beta de $20 \%$ e erro alfa de 5\%, seriam necessárias 364 gestantes para esta avaliação.

Dados da anamnese (idade, gesta, partos, abortos, peso dos recém-nascidos) foram anotados, bem como dados do exame físico (medida da altura uterina, batimentos cardíacos fetais, peso materno prévio e peso materno na primeira consulta), bem como avaliação ultrassonográfica do crescimento fetal realizada em todos os trimestres. Para a avaliação da altura uterina a paciente foi colocada em decúbito dorsal, com a bexiga vazia, utilizando fita métrica graduada em centímetros, da borda superior do pube ao fundo uterino.

As consultas foram realizadas mensalmente até a $36^{a}$ semana de gestação e semanalmente até o parto. Em todas as consultas, a medida da altura uterina foi realizada e anotada. As medidas das alturas uterinas foram agrupadas em intervalos de quatro semanas, considerando que as consultas até 36 semanas foram realizadas mensalmente. A escolha desse intervalo foi convencionada pelos pesquisadores, do seguinte modo: $16-20$ semanas (4으ês); $\geq 20-24$ semanas (5o mês); $\geq 24-28$ semanas (6o mês); $\geq 28-32$ semanas (7은 mês); $\geq 32-36$ semanas ( 8 o mês) e $\geq 36$ semanas (9o mês). Quando a paciente teve mais de uma medida neste intervalo, foi considerada a média dessas medidas naquele intervalo.

Na ultrassonografia utilizou-se a sonda convexa de $3,75 \mathrm{mHz}$, normalmente adotada para essa avaliação. A paciente foi colocada em decúbito dorsal, para que as medidas pudessem ser realizadas. A fórmula utilizada foi a descrita Hadlock com erro estimado em 10\%, expresso em gramas exatas, se baseia na circunferência abdominal $(C A)$, circunferência cefálica $(C C)$, comprimento do fêmur (CF), diâmetro biparietal 
(DBP), comprimento do fêmur (CF) em uma equação logarítmica. As medidas do peso fetal também foram agrupadas de acordo com as semanas gestacionais, do seguinte modo: $16-20$ semanas (40 mês); $\geq 20-24$ semanas ( $5 \circ$ mês); $\geq 24-28$ semanas ( $6 \circ$ mês); $\geq 28-32$ semanas (7o mês); $\geq 32-36$ semanas ( 8 o mês) e $\geq 36$ semanas (9o mês) (BLUE NR, et al., 2018).

Foram incluídas gestantes de baixo risco de ambos os serviços e que permitiram que seus dados fossem utilizados através da assinatura do termo de consentimento livre e esclarecido (TCLE). Foram excluídas gestantes que não permitiram que seus dados fossem avaliados, as que não concordaram com o protocolo dos serviços em questão, gestantes de alto risco obstétrico, aquelas cujos dados estavam incompletos e aquelas com intercorrências durante o pré-natal (anemia, infecção, sangramento, parasitose intestinal) e adolescentes. A idade maior que 35 anos não foi fator de exclusão, se não houvesse comorbidade associada ou intercorrência clínica no pré-natal.

Os dados foram digitados diretamente em planilha Excel e depois exportados para o software Jamovi. Foram comparadas as alturas uterinas para os intervalos de idade gestacional e sua associação com dados clínicos e epidemiológicos. Os testes estatísticos utilizados foram a análise da variância (ANOVA) para comparação de médias entre grupos ou teste qui-quadrado para variáveis categóricas ou, quando necessário, o teste de Kruskall Wallis (Teste H). O nível de significância foi $p<0,05$. O presente estudo foi aprovado pelo Comitê de Ética, via Plataforma Brasil, sob o número 3.489.723.

\section{RESULTADOS}

Foram estudadas 883 gestantes oriundas do serviço de baixo risco obstétrico, sem qualquer comorbidade associada. A média de idade das pacientes estudadas foi de $25,5 \pm 7,29$, sendo a maior idade de pré-natal 45 anos. A maioria das pacientes apresentou em gestação anterior recém-nascidos considerados adequados para a idade gestacional (AIG), com peso entre 2500 e $4000 \mathrm{~g}$. A frequência de macrossômicos foi baixa, nessa amostra (3,10\%). Os demais dados epidemiológicos e obstétricos são apresentados na Tabela 1.

Tabela 1 - Dados obstétricos e epidemiológicos das gestantes estudadas.

\begin{tabular}{lcc}
\hline Dados avaliados & Média \pm Desvio padrão & Mediana \\
\hline Dados obstétricos & & \\
\hline Gesta & $2,21 \pm 1,55$ & 2,00 \\
Partos & $0,97 \pm 1,31$ & 1,00 \\
Abortos & $0,24 \pm 0,57$ & 0,00 \\
Cesariana anterior & $0,29 \pm 0,63$ & 0,00 \\
Parto vaginal anterior & $0,68 \pm 1,25$ & 0,00 \\
Peso na primeira consulta & $70,90 \pm 17,8$ & 67,20 \\
Peso prévio & $65,90 \pm 15,90$ & 63,00 \\
\hline Peso do recém-nascido em & $\mathbf{N}$ & $\%$ \\
gestações anteriores & 78 & 8,70 \\
\hline$<2500$ g & 342 & 38,30 \\
$\geq 2500$ g e $<4000 g$ & 28 & 3,10 \\
$\geq 4000$ g & $\mathbf{N}$ & $\%$ \\
\hline Dados epidemiológicos & 96 & 10,80 \\
\hline Tabagismo & 75 & 8,40 \\
Etilismo & 10 & 1,10 \\
Drogas & Média & Mediana \\
\hline Renda familiar (Reais) & 1379,00 & Desvio padrão \\
\end{tabular}

Fonte: Assis GP, et al., 2021.

Quando se avaliou as alturas uterinas identificaram-se os seguintes resultados médios (percentil 50) para cada intervalo de altura uterina: 17,0 $\pm 2,59$ (16-20 semanas; 4o mês), 21,0 $\pm 2,27$ (20-24 semanas; 5o mês), 24,9 $\pm 2,45$ ( $24-28$ semanas; 6 o mês), 28,4 $\pm 2,49$ (28-32 semanas; 7o mês), 32,00 \pm 2,26 (32-36 semanas; 8 o mês) e $34,00 \pm 12,00$ ( $\geq 36$ semanas; 9 o mês). Na Figura 2 são apresentados os percentis para cada intervalo de idade gestacional. 
Figura 2 - Percentis para a altura uterina em $\mathrm{cm}$ avaliado em gestantes de baixo risco de acordo com intervalos da idade gestacional.

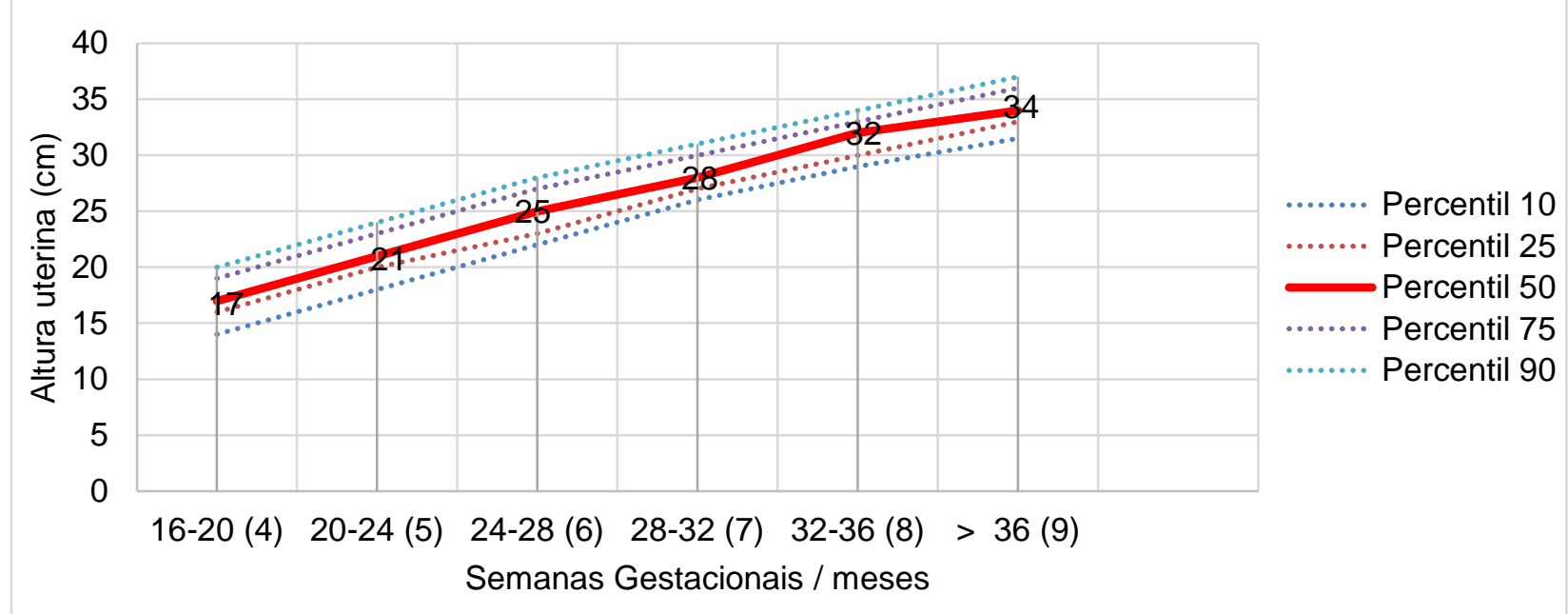

Legenda: A linha sólida representa o percentil 50. No eixo $X$ são as semanas gestacionais e no eixo $Y$, a altura uterina.

Fonte: Assis GP, et al.,2021.

A avaliação do peso estimado pelo ultrassom ao longo do pré-natal foi também distribuída através de percentis, conforme Figura 3. Os percentis utilizados foram 10,25,50,75 e 90.

Figura 3 - Percentis de peso fetal em gramas estimado ao ultrassom para as idades gestacionais.

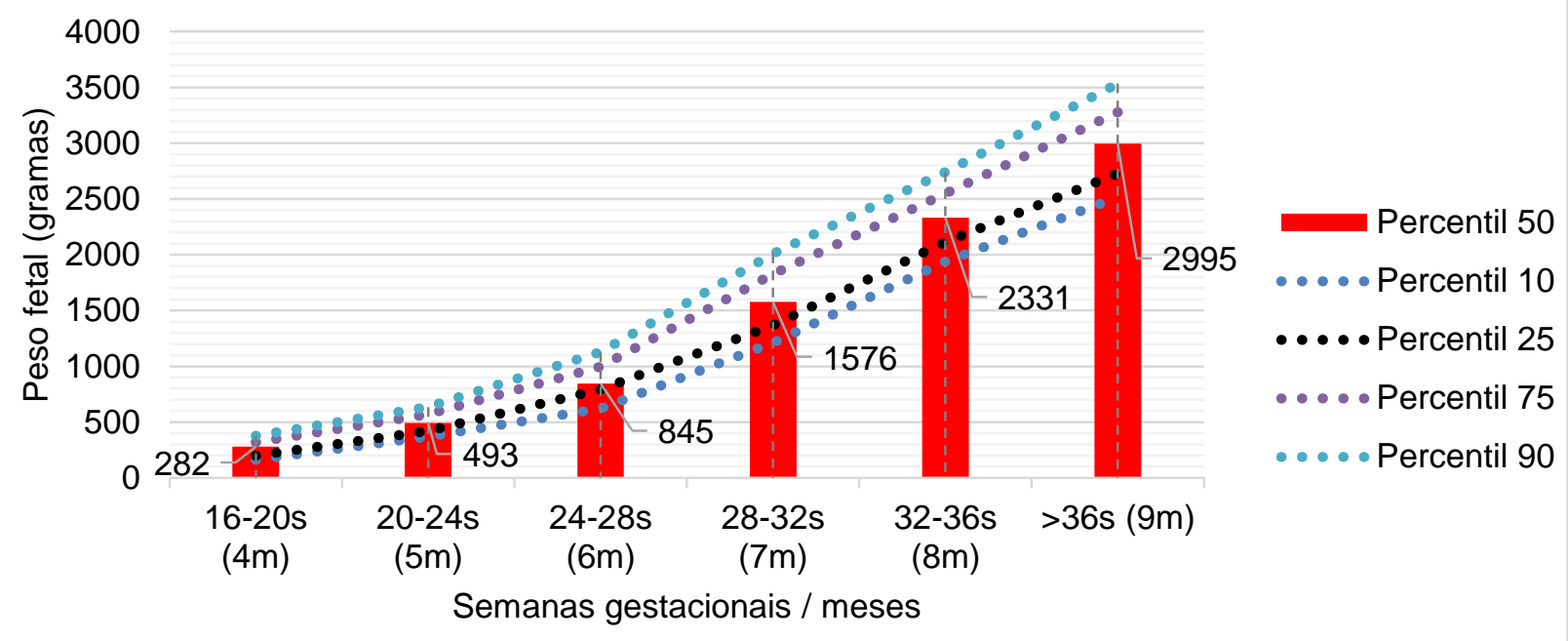

Legenda: As barras vermelhas representam o percentil 50 para cada idade gestacional. As linhas coloridas os demais percentis.

Fonte: Assis GP, et al., 2021.

O peso fetal não foi associado ao tabagismo ou ao uso de drogas ( $p>0,05)$. O peso fetal $\geq 36$ semanas não foi associado à idade, renda familiar, peso materno na primeira consulta e peso materno prévio, utilizando a correlação e regressão linear $(p>0,05)$, mas foi associado à altura uterina, de forma que quanto maior a altura uterina, maior o peso. Realizou-se análise multivariada buscando avaliar os fatores associados pelo peso fetal acima de 36 semanas, mas o único dado que mostrou associação positiva foi a altura uterina $(p<0,001)$, sendo que o peso prévio materno, peso materno na primeira consulta e histórico do peso do recémnascido anterior não foi associado ao peso fetal no último mês de gestação ( $p>0,05)$. Na Tabela 2 são apresentadas as medidas de altura uterina e peso fetal para cada intervalo de idade gestacional e o percentil avaliado. 
Tabela 2 - Medidas de altura uterina e peso fetal para cada intervalo de idade gestacional.

\begin{tabular}{cccccccccccc}
\hline \multirow{2}{*}{ IG Sem } & \multirow{2}{*}{ IG Mês } & $\begin{array}{c}\text { Peso } \\
\mathbf{1 0}\end{array}$ & $\begin{array}{c}\text { AU } \\
\mathbf{1 0}\end{array}$ & $\begin{array}{c}\text { Peso } \\
\mathbf{2 5}\end{array}$ & $\begin{array}{c}\text { AU } \\
\mathbf{2 5}\end{array}$ & $\begin{array}{c}\text { Peso } \\
\mathbf{5 0}\end{array}$ & $\begin{array}{c}\text { AU } \\
\mathbf{5 0}\end{array}$ & $\begin{array}{c}\text { Peso } \\
\mathbf{7 5}\end{array}$ & $\begin{array}{c}\text { AU } \\
\mathbf{7 5}\end{array}$ & $\begin{array}{c}\text { Peso } \\
\mathbf{9 0}\end{array}$ & $\begin{array}{c}\text { AU } \\
\mathbf{9 0}\end{array}$ \\
\hline $16-20$ & 4 & 162 & 14 & 199 & 16 & 282 & 17 & 318 & 19 & 376 & 20 \\
$20-24$ & 5 & 371 & 18 & 418 & 20 & 491 & 21 & 568 & 23 & 634 & 24 \\
$\lg 24-28$ & 6 & 622 & 22 & 703 & 23 & 843 & 25 & 996 & 27 & 1127 & 28 \\
$\lg 28-32$ & 7 & 1211 & 26 & 1370 & 27 & 1576 & 28 & 1828 & 30 & 2011 & 31 \\
$\lg 32-36$ & 8 & 1942 & 29 & 2115 & 30 & 2332 & 32 & 2550 & 33 & 2742 & 34 \\
$\lg >36$ & 9 & 2526 & 31,5 & 2735 & 33 & 2995 & 34 & 3274 & 36 & 3534 & 37 \\
\hline
\end{tabular}

Legenda: AU: Altura uterina; $10,25,50,75,90$ - percentis. IG: idade gestacional.

Fonte: Assis GP, et al., 2021.

Abaixo, encontra-se a curva de normalidade considerando o peso e a altura uterina em cada intervalo gestacional selecionado, em meses (Figura 4). A interpretação do gráfico deve ser assim realizada: Para uma idade gestacional de 4 meses, a altura uterina média é de $15 \mathrm{~cm}$ e o peso fetal médio de $282 \mathrm{~g}$ e assim sucessivamente.

Figura 4 - Curva da altura uterina para o intervalo de idade gestacional.

40

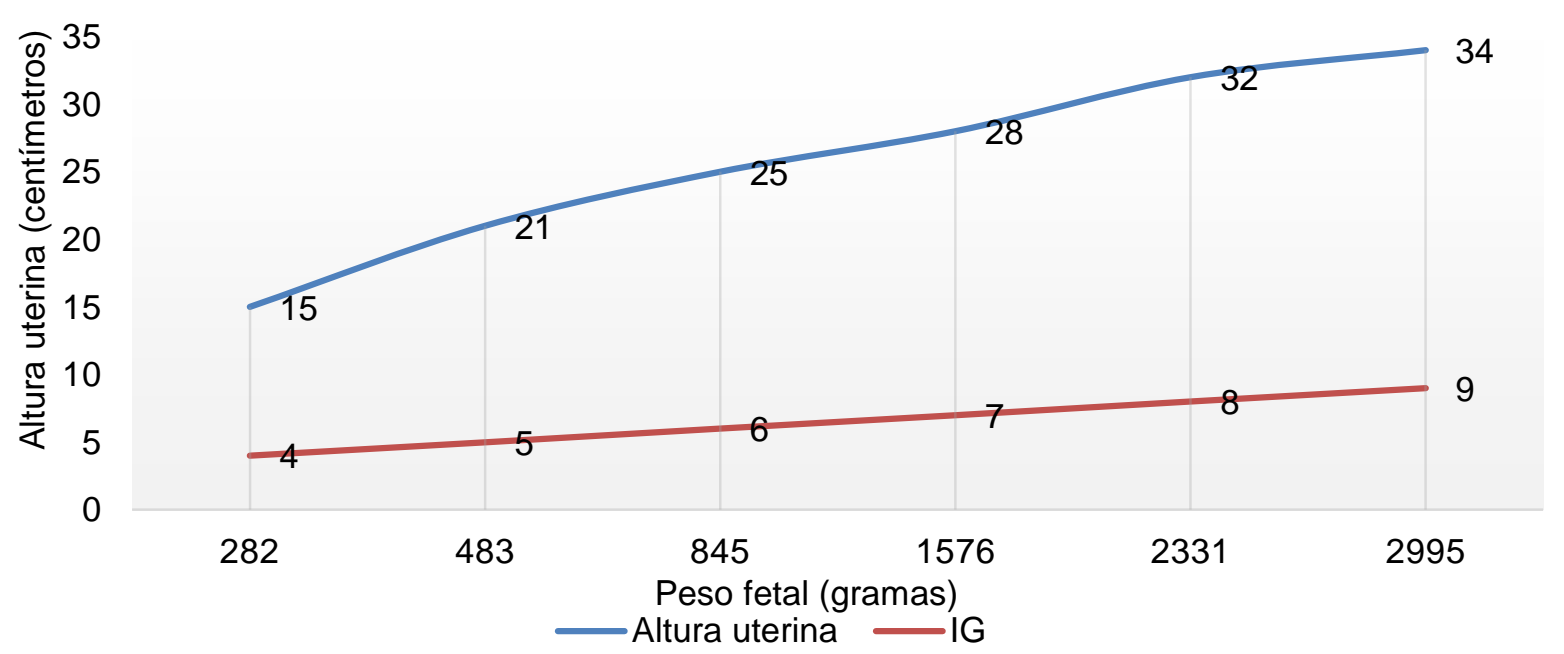

Legenda: Altura uterina (em $\mathrm{cm}-\mathrm{Y})$, peso (em $\mathrm{g}-\mathrm{X})$ para o intervalo de idade gestacional (em meses - $\mathrm{Y}$ ).

Fonte: Assis GP, et al., 2021.

\section{DISCUSSÃO}

A avaliação dos dados clínicos e epidemiológicos nos permitiu identificar que a maioria das pacientes eram jovens, na segunda gestação, com parto vaginal anterior, não tabagista e não etilista, com média de renda compatível com o salário-mínimo do Brasil. Essa população representa a grande maioria da população brasileira estudada durante a gestação (MARTINELLI S, et al., 2011; FREIRE DMC, et al., 2006).

Foi proposta a criação de uma curva de normalidade para a altura uterina, em função da idade gestacional e peso fetal estimado pelo ultrassom, em gestantes com idade gestacional confiável, de baixo risco obstétrico, sem fatores associados para os desvios de crescimento, para auxiliar às estimativas do peso fetal na população brasileira, reduzindo os custos do pré-natal, sem prejuízos assistenciais. Interessante que poucos foram os estudos realizados comparando o peso fetal, idade gestacional e a altura uterina. Apesar de antigos, são estudos consagrados na literatura médica, mas não refletem necessariamente a população brasileira, uma vez que foram avaliadas gestantes de outros países (BELIZAN J, et al., 1978; POMMIER M, et al., 1979; QUARANTA P, et al., 1981) e até mesmo a curva utilizada no Brasil, pelo Ministério da Saúde, reflete uma amostra da população uruguaia (BRASIL, 2012). 
$\mathrm{Na}$ maioria, os estudos nacionais existentes buscaram associar duas variáveis (altura uterina e idade gestacional ou peso fetal e idade gestacional) (MARTINELLI S, et al., 2001). Cecatti JG, et al. (2000) estudando 2.874 gestantes para o estabelecimento de uma curva de normalidade para o peso fetal estimado por ultrassonografia (considerando a idade gestacional) demonstraram a importância de ser realizada uma curva com participantes brasileiras, abrangendo a realidade socioeconômica e características demográficas do país.

Importante ressaltar que os estudos nacionais que buscaram comparar a altura uterina com a idade gestacional possuem muitas divergências, principalmente devido à diferentes metodologias - critérios de inclusão, técnica de medida da altura uterina, múltiplos examinadores, inclusão de população de risco obstétrico e até o próprio biótipo da gestante que poderia determinar vieses. Neste estudo, foram padronizadas as medidas, sendo um mesmo pesquisador que conferiu todas as medidas uterinas, o que minimizou as diferenças de medidas entre observadores. Além disso, a população escolhida foi a de baixo risco obstétrico, para reduzir o impacto da patologia obstétrica no crescimento uterino. (CECATTI JG, et al, 2000).

Apesar dos estudos descreverem a altura uterina semanal, a comparação com intervalo de quatro semanas desse estudo foi possível, construindo o intervalo a partir dos dados semanais. Comparando com os dados de Freire DMC, et al. (2006), Martinelli S, et al. (2011) e de Barini R (1997) os dados foram muito semelhantes, conforme descrito na Tabela 3. Dessa forma, apesar do Brasil ser um país heterogêneo, com características diferentes, os parâmetros de crescimento fetal se mostraram semelhantes comparando as curvas brasileiras feitas em regiões distintas, com populações diferentes, o que mostra uma tendência do crescimento fetal em gestantes brasileiras de baixo risco.

Por outro lado, a comparação com a curva utilizada pelo MS mostrou-se bem diferente, já que foi construída a partir de uma amostra de mulheres uruguaias. Tais dados são compatíveis estudo brasileiro que identificou que para a detecção de desvios do crescimento fetal, especialmente, PIG, a sensibilidade da curva brasileira de Freire DMC, et al. (2006) foi $51,6 \%$, enquanto a sensibilidade da curva uruguaia foi de $12,5 \%$, talvez porque ela não seja a melhor ferramenta para acompanhamento de nossas gestantes (PAIVA DMC, 2006).

Tabela 3 - Comparação entre os valores de altura uterina para a idade gestacional. Observa-se que para essa comparação foram utilizados os valores das alturas uterinas entre as idades gestacionais selecionadas.

\begin{tabular}{|c|c|c|c|c|c|c|c|c|c|c|c|c|c|c|c|c|}
\hline \multirow[t]{2}{*}{ IG } & \multicolumn{5}{|c|}{$\begin{array}{l}\text { Valores de altura uterina } \\
\text { considerando os percentis desse } \\
\text { estudo (ASSIS GP, et al., 2021) }\end{array}$} & \multicolumn{5}{|c|}{$\begin{array}{l}\text { Valores da altura uterina para cada } \\
\text { percentil (FREIRE DMC, et al., } \\
\text { 2006). }\end{array}$} & \multicolumn{3}{|c|}{$\begin{array}{l}\text { Valores da altura } \\
\text { uterina para cada } \\
\text { percentil } \\
\text { (MARTINELLI S. et } \\
\text { al., 2001). }\end{array}$} & \multicolumn{3}{|c|}{$\begin{array}{l}\text { Valores da altura } \\
\text { uterina para cada } \\
\text { percentil (BARINI } \\
\text { R.,1997) }\end{array}$} \\
\hline & $\mathrm{P}^{10}$ & $\mathrm{P}^{25}$ & $\mathrm{P}^{50}$ & $\mathrm{P}^{75}$ & $\mathrm{P}^{90}$ & $P^{10}$ & $\mathrm{P}^{25}$ & $\mathrm{P}^{50}$ & $P^{75}$ & $\mathrm{P}^{90}$ & $\mathrm{P}^{10}$ & $P^{50}$ & $\mathrm{P}^{90}$ & $\mathrm{P}^{10}$ & $\mathrm{P}^{50}$ & $\mathrm{P}^{90}$ \\
\hline$\geq 16$ & \multirow{2}{*}{14} & \multirow{2}{*}{16} & \multirow{2}{*}{17} & \multirow{2}{*}{19} & \multirow{2}{*}{20} & 12,5 & 13,5 & 15,2 & 16,8 & 18,5 & & & & & & \\
\hline$<20$ & & & & & & 15,0 & 16,8 & 18,5 & 19,5 & 21,0 & & & & & & \\
\hline$\geq 20$ & \multirow{2}{*}{18} & \multirow{2}{*}{20} & \multirow{2}{*}{21} & \multirow{2}{*}{23} & \multirow{2}{*}{24} & 16,3 & 17,5 & 19,2 & 20,5 & 21,9 & 18,8 & 20,7 & 22,6 & 15 & 18 & 21,2 \\
\hline$<24$ & & & & & & 19,7 & 21,1 & 22,2 & 24,0 & 25,7 & 21,5 & 23,4 & 25,3 & 18 & 21 & 24 \\
\hline$\geq 24$ & \multirow{2}{*}{22} & \multirow{2}{*}{23} & \multirow{2}{*}{25} & \multirow{2}{*}{27} & \multirow{2}{*}{28} & 20,9 & 22,1 & 22,8 & 24,8 & 27,4 & 22,4 & 24,2 & 25,3 & 18 & 22 & 25 \\
\hline$<28$ & & & & & & 23,5 & 25,0 & 26,0 & 27,0 & 27,4 & 24,8 & 26,7 & 28,8 & 22 & 25 & 28 \\
\hline$\geq 28$ & \multirow{2}{*}{26} & \multirow{2}{*}{27} & \multirow{2}{*}{28} & \multirow{2}{*}{30} & \multirow{2}{*}{31} & 25,0 & 25,5 & 26,6 & 27,5 & 28,0 & 25,6 & 27,5 & 29,4 & 23 & 26 & 28 \\
\hline$<32$ & & & & & & 26,1 & 27,8 & 28,8 & 30,7 & 31,8 & 27,8 & 29,7 & 31,6 & 25 & 28 & 31 \\
\hline$\geq 32$ & \multirow{2}{*}{29} & \multirow{2}{*}{30} & \multirow{2}{*}{32} & \multirow{2}{*}{33} & \multirow{2}{*}{34} & 27,3 & 28,8 & 29,8 & 31,2 & 31,6 & 28,5 & 30,4 & 32,5 & 26 & 29 & 32 \\
\hline$<36$ & & & & & & 29,7 & 30,9 & 32,0 & 33,0 & 34,2 & 30,4 & 32,3 & 34,2 & 29 & 32 & 35 \\
\hline \multirow{2}{*}{$\geq 36$} & \multirow{2}{*}{$\begin{array}{c}31 \\
5\end{array}$} & \multirow{2}{*}{33} & \multirow{2}{*}{34} & 36 & 37 & 31 & 31,9 & 33,0 & 33,0 & 34,2 & 31,1 & 32,9 & 34,8 & 30 & 32 & 35 \\
\hline & & & & 30 & $3 r$ & 32,5 & 33,0 & 34,2 & 36,4 & 37,2 & 32,8 & 34,7 & 36,6 & 31 & 34 & 38 \\
\hline
\end{tabular}

Fonte: Assis GP, et al., 2021.

Quando se avaliou os elementos responsáveis pelo peso fetal, o único dado relevante em nossa análise foi a altura uterina $(p<0,05)$. O peso materno prévio, o peso inicial, o ganho de peso e o peso final materno não tiveram associação com o crescimento uterino, representado pela avaliação da altura uterina. Dessa 
forma, é lícito afirmar que a altura uterina é uma boa medida para a estimativa do peso fetal, em gestantes de baixo risco (DONGOL A, et al., 2020; BELETE W e GAYM A., 2008). Apesar de alguns estudos associarem o peso materno ao peso fetal, esses achados não foram replicados nesse estudo, talvez porque a amostra foi de pacientes de baixo risco ou porque o pré-natal eficiente foi um bom modulador, evitando o ganho exagerado de peso (NOMURA RMY, et al, 2012).

Outro estudo com pacientes nigerianas, identificou que o método clínico (medida da altura uterina e altura da apresentação fetal) superestimou o peso ao nascer, enquanto o ultrassom o subestimou. O erro percentual absoluto médio do método clínico foi menor do que o do método ultrassonográfico, mas sem significância estatística, para fetos entre 2500 e 4000g (SHITTU AS, et al., 2007).

Dessa forma, especula-se que a estimativa clínica do peso ao nascer seja tão precisa quanto a estimativa ultrassonográfica de rotina, exceto em bebês com baixo peso ao nascer. Portanto, quando o método clínico sugere peso menor do que $2.500 \mathrm{~g}$, a avaliação ultrassonográfica subsequente é recomendada para produzir uma melhor previsão e avaliar o bem-estar fetal (SHITTU AS, et al., 2007).

Entretanto, a avaliação clínica da altura uterina não pode ser negligenciada. Pelo contrário, ela traz importantes informações em relação ao peso fetal, devendo ainda ser encorajada na prática obstétrica atual, especialmente em países pobres. Apesar deste estudo não verificar a validade dessa curva para predizer os desvios de crescimento fetal, porque apenas trabalhamos com gestantes de baixo risco, a curva criada é de fácil aplicação, baixo custo e prática, por ser mensal e não semanal, além de iniciar com 16 semanas, sendo uma ferramenta útil na consulta obstétrica de rotina, em gestantes de baixo risco, já que associou peso fetal, idade gestacional e altura uterina (MARTINELLI S, et al., 2001; FREIRE DMC, et al., 2006).

A curva criada neste estudo poderá ajudar na condução de pré-natais em muitas regiões do país. A curva mensal, facilita a avaliação por enfermeiros e médicos que atendem baixo risco, já que o Ministério de Saúde, propõe consultas mensais até 36 semanas de gestação (BRASIL, 2000; BRASIL, 2012). Como em muitas regiões desse país a ultrassonografia não está disponível de forma rotineira, a avaliação do crescimento fetal através de ferramentas práticas, favorecem o atendimento, sem incrementar os custos, podendo a ultrassonografia ser realizada quando as medidas identificadas estiverem fora dos parâmetros propostos pela curva criada nesse estudo. Sugere-se novos estudos para a validação dessa curva nos desvios de crescimento fetal.

\section{CONCLUSÃO}

Baseado no exposto, identificou-se que a curva de altura uterina $\mathrm{x}$ peso fetal $\mathrm{x}$ idade gestacional pode ser uma ferramenta de uso em acompanhamento de gestantes de baixo risco, já que o peso fetal aumenta com a idade gestacional. Os resultados apontam que não houve associação do peso fetal com o peso materno prévio, com o peso materno no início do pré-natal ou com o ganho de peso materno, o que encoraja a utilizar o parâmetro de crescimento uterino como medida indireta do peso fetal. Dessa forma, é lícito afirmar que a altura uterina é uma boa medida para a estimativa do peso fetal, em gestantes de baixo risco. Sendo assim, a curva criada nesse estudo poderá ter aplicabilidade prática no acompanhamento dessas gestantes.

\section{REFERÊNCIAS}

1. ANTONELLI JDS, et al. As consequências da restrição de crescimento intrauterino na estrutura e fluxo sanguíneo cerebral: uma revisão da literatura. Femina®, 2018; 46(6): 352-359

2. BARINI R. Avaliação da curva de crescimento da altura uterina como método para estimar o peso fetal. Tese. (Doutorado). Universidade Estadual de Campinas; 1989. Disponível em http://repositorio.unicamp.br/bitstream/REPOSIP/308218/1/Barini_Ricardo_D.pdf. Acesso em 10 maio 2021.

3. BELETE W, GAYM A. Clinical estimation of fetal weight in low resource settings: comparison of Johnson's formula and the palpation method. Ethiop Med J, 2008; 46(1): 37-46.

4. BELIZÁN J, et al. Diagnosis of intrauterine growth retardation by a simple clinical method: measurement of uterine height. Am J Obstet Gynecol,1978; 131: 643-6.

5. BLUE NR, et al. The Hadlock Method Is Superior to Newer Methods for the Prediction of the Birth Weight Percentile. Journal of Ultrasound in Medicine, 2018; 38(3): 587-96. 
6. BRASIL. Ministério da Saúde. Cadernos de Atenção Básica. Atenção ao pré-natal de baixo risco. 2012. 320p. Disponível em http://bvsms.saude.gov.br/bvs/publicacoes/cadernos_atencao_basica_32_prenatal.pdf. Acesso em 10 abril 2021.

7. BRASIL. MINISTÉRIO DA SAÚDE DO BRASIL. Assistência pré-natal. 2000. 68p. Disponível em https://bvsms.saude.gov.br/bvs/publicacoes/cd04_11.pdf. Acesso em 10 abril 2021

8. CALDERON IMP, RUDGE MVC. Macrossomia fetal: um desafio obstétrico. Revista Brasileira de Ginecologia e Obstetrícia, 2006; 28(4): 211-13.

9. CECATTI JG, et al. Curva dos valores normais de peso fetal estimado por ultra-sonografia segundo a idade gestacional. Cad. Saúde Pública, 2000; 16(4): 1083-1090.

10. CROCKART IC, et al. Classification of intrauterine growth restriction at 34-38 weeks' gestation with machine learning models. Inform Med Unlocked. 2021; 23: 100533.

11. CURTI A, et al. Multivariable evaluation of term birth weight: a comparison between ultrasound biometry and symphysis-fundal height. J Matern Fetal Neonatal Med, 2014; 27(13): 1328-32.

12. DETER RL, et al. Third-trimester growth diversity in small fetuses classified as AGA or SGA at birth. Ultrasound Obstet Gynecol. 2021; 16 (5). Disponível em https://obgyn.onlinelibrary.wiley.com/doi/10.1002/uog.23688. Acesso em 10 abril 2021.

13. DONGOL A, et al. Clinical Estimation of Fetal Weight with Reference to Johnson's Formula: An Alternative Solution Adjacent to Sonographic Estimation of Fetal Weight. Kathmandu University Medical Journal, 2020; 18(2): 7-12.

14. FEBRASGO. Federação Brasileira de Ginecologia e Obstetrícia.

15. FREIRE DMC, et al. A altura uterina é capaz de diagnosticar os desvios do volume de líquido amniótico? Rev Bras Ginecol Obstet, 2013; 35(2): 49-54.

16. FREIRE DMC, et al. Curva da altura uterina por idade gestacional em gestantes de baixo risco. Rev Bras Ginecol Obstet, 2006; 28(1): 3-9.

17. GONZÁLEZ-FERNANDES D, et al. Identifies High Prevalence of Low Symphysis-Fundal Height in Indigenous Pregnant Women Experiencing Multiple Infections, Nutrient Deficiencies, and Inflammation: The Maternal Infections, Nutrient Deficiencies, and Inflammation (MINDI) Cohort. Current Developments in Nutrition, 2021; 5(4): 1-16.

18. MARTINELLI S, et al. Proposta de Nova Curva de Altura Uterina para Gestações entre a $20^{\underline{a}}$ e a $42^{\underline{a}}$ Semana. Rev. Bras. Ginecol. Obstet. 2001, 23(4): 235-41

19. MOREIRA NETO AR, et al. Etiologia da restrição de crescimento intrauterino (RCIU). Com. Ciências Saúde, 2011; 22(1): 21-30.

20. NOMURA RMY et al. Influência do estado nutricional materno, ganho de peso e consumo energético sobre o crescimento fetal, em gestações de alto risco. Rev. Bras. Ginecol. Obstet. 2012; 34(3): 107-112.

21. PAIVA DMC. Validação da curva de altura uterina para rastreamento dos desvios de crescimento fetal. Faculdade de Ciências Médicas da Universidade Estadual de Campinas. Tese. (Tocoginecologia). 2006.114p. Disponível em http://repositorio.unicamp.br/bitstream/REPOSIP/312599/1/Paiva_DjacyrMagnaCabral_D.pdf.

22. POMMIER M. Estudio de la altura uterina del fondo uterino para la detección del crecimiento intrauterino retardado. Ginecol Obstet Mex, 1979; 46(276): 253-9.

23. QUARANTA P, et al. Prediction of small-for-dates infants by measurement of symphysial-fundal-height. Br $\mathrm{J}$ Obstet Gynaecol, 1981; 88: 115-9.

24. ROBERT PETER J, et al. Symphysial fundal height (SFH) measurement in pregnancy for detecting abnormal fetal growth. Cochrane Database Syst Rev, 2015; (9):CD008136.

25. SHARP A, et al. Screening and management of the small for gestational age fetus in the UK: A survey of practice. Eur J Obstet Gynecol Reprod Biol, 2018; 231: 220-224.

26. SHITTU AS, et al. Clinical versus sonographic estimation of fetal weight in southwest Nigeria. J Health Popul Nutr, 2007; 25(1): 14-23.

27. TAYLOR P, et al. Symphysial-fundal height from 12 weeks' gestation. Aust N Z J Obstet Gynaecol, 1984; $24: 189-91$.

28. ZAHRAN M, et al. Analysis of the effectiveness of ultrasound and clinical examination methods in fetal weight estimation for term pregnancies. Turk J Obstet Gynecol, 2015; 12(4): 220-225.

29. ZIMMERMMANN JB, et al. Condutas em obstetrícia para gestantes de baixo risco. Protocolo do serviço de Obstetrícia da Faculdade de Medicina da UFJF. 2018; 350p. 